\title{
Availability of irinotecan in a second-line setting confers survival benefit to patients with advanced gastric cancer refractory to fluoropyrimidine-based regimens
}

\author{
MASARU OBA, KEISHO CHIN, YOSHIMASA KAWAZOE, KOICHI TAKAGI, MARIKO OGURA, EIJI SHINOZAKI, \\ MITSUKUNI SUENAGA, SATOSHI MATSUSAKA, NOBUYUKI MIZUNUMA and KIYOHIKO HATAKE
}

Gastrointestinal Group, Division of Medical Oncology, Cancer Institute Hospital

of the Japanese Foundation for Cancer Research, Tokyo, Japan

Received November 17, 2010; Accepted January 18, 2011

DOI: $10.3892 / \mathrm{ol} .2011 .241$

\begin{abstract}
Optimal second-line chemotherapy may contribute to favorable survival in patients who receive first-line treatment for advanced gastric cancer. The aim of this retrospective study was to compare a second-line setting using irinotecan with paclitaxel in terms of survival benefit and safety. A total of 179 patients with recurrent or unresectable gastric cancer who had received prior chemotherapy with a fluoropyrimidine-based regimen were treated with irinotecan alone at $150 \mathrm{mg} / \mathrm{m}^{2}$ on days 1 and 15 every 4 weeks (Cohort I) or weekly paclitaxel at $80 \mathrm{mg} / \mathrm{m}^{2}$ on days 1,8 and 15 every 4 weeks (Cohort P) between April, 2004 and March, 2009. Patient characteristics, overall response rate, disease control rate, progression-free survival, overall survival and safety were investigated. Of the 179 patients, 92 received irinotecan and 87 patients who were contraindicated for irinotecan received weekly paclitaxel. The overall response and disease control rates in Cohort I were 6.5 and $43.5 \%$, respectively, as compared with 9.8 and $54.9 \%$, respectively, in Cohort $\mathrm{P}$. No variation was noted in median progression-free survival (Cohort I vs. P, 2.6 vs. 2.8 months; $\mathrm{P}=0.812$ ), whereas median overall survival (Cohort I vs. P, 9.8 vs. 4.9 months; $\mathrm{P}<0.0001)$ differed significantly between the two cohorts. The most common grade $3 / 4$ adverse events were neutropenia, leukopenia, anemia and anorexia, which were tolerable in each treatment cohort. Availability of irinotecan in a second-line setting confers a survival benefit to advanced gastric cancer patients in whom fluoropyrimidine-based firstline chemotherapy was unsuccessful.
\end{abstract}

Correspondence to: Dr Masaru Oba, Division of Medical Oncology, Cancer Institute Hospital of the Japanese Foundation for Cancer Research, 3-8-31 Ariake, Koto-ku, Tokyo 135-8550, Japan E-mail: masaru.oba@jfcr.or.jp

Key words: gastric cancer, irinotecan, paclitaxel, second-line chemotherapy

\section{Introduction}

Gastric cancer (GC) is one of the most common types of cancer in East Asia and the second leading cause of cancerrelated death worldwide, with over 730,000 deaths per year (1). Although noteworthy advances in the early diagnosis and endoscopic or surgical resection of this entity have occurred in recent years in Japan and Korea, advanced stage is achieved prior to detection when curative resection may no longer be a viable option. When GC is advanced, systemic chemotherapy is regarded as crucial in improving patient quality of life and prolonging survival (2-4). However, GC causes a deficiency in nutrition due to peritoneal metastasis, rapidly depriving patients of their well-being and favorable performance status (PS). It is difficult to control this entity by first-line chemotherapy alone, despite the availability of numerous cytotoxic agents that are active against GC. Currently, a 5-fluorouracil (FU)-based regimen in combination with cisplatin (CDDP) is the most commonly used first-line therapy in the treatment of advanced GC (5-7). However, the optimal regimen for a second-line chemotherapy for GC remains to be determined. Although the SPIRITS trial reported an overall survival (OS) of more than 1 year, progression-free survival (PFS) with S-1 plus CDDP in a first-line setting was only 6 months, and $74 \%$ of the patients received second-line chemotherapy (8). Moreover, in the JCOG9912 trial, OS and PFS were 11.4 and 4.2 months, respectively, in the S-1 alone arm, and 74\% of the patients received second-line chemotherapy (9). By contrast, in the FLAGS global trial, OS and PFS were 8.6 and 4.8 months, respectively, in the S- 1 plus CDDP arm, and only $31 \%$ of the patients received second-line chemotherapy (10). In the V-325 trial (6), OS and time-to-progression were 9.2 and 5.6 months, respectively, in the DCF (docetaxel, CDDP and FU) arm, and only $32 \%$ of the patients received further chemotherapy. This indicates that, in certain cases, optimal second-line chemotherapy may contribute to the favorable OS observed with first-line treatment.

Recently, Thuss-Patience et al reported that irinotecan (CPT-11) alone $\left(250 \mathrm{mg} / \mathrm{m}^{2}\right.$ every 3 weeks, to be increased to $350 \mathrm{mg} / \mathrm{m}^{2}$ depending on toxicity) significantly improved OS compared to best supportive care $[\mathrm{HR}=0.48 ; 95 \%$ confidence 
interval $(\mathrm{CI}), 0.25-0.92 ; \mathrm{P}=0.023$ ] in a second-line setting in patients previously treated with only one regimen (11). Consequently, CPT-11 may be a key novel agent for the treatment of advanced GC in a second-line setting. On the other hand, the weekly administration of paclitaxel (PAC) for $\mathrm{GC}$ following the failure of prior chemotherapy has become standard practice in Japan, as it offers milder toxicities than a tri-weekly schedule but with equivalent activity $(12,13)$. In previous phase II trials, it was found that PAC was active against $\mathrm{GC}$ with peritoneal metastasis that was refractory to regimens containing 5-FU $(14,15)$. However, only a few phase II trials involving weekly PAC regimen for GC have been reported in a second-line setting.

At our institute, CPT-11 is the preferred agent in a secondline setting, unless contraindicated due to peritoneal metastasis or inadequate liver function, in which case taxane agents are selected. The purpose of the present retrospective study was to compare CPT-11 alone and weekly PAC in a second-line setting following the failure of a fluoropyrimidine-based regimen in terms of survival benefit and safety in the treatment of recurrent or unresectable GC.

\section{Patients and methods}

Patients. Between April, 2004 and March, 2009, 179 patients received second-line chemotherapy with CPT-11 monotherapy or weekly PAC at Division of Medical Oncology, Cancer Institute Hospital of the Japanese Foundation for Cancer Research, Tokyo, Japan. Of these, 92 patients with no findings of peritoneal metastasis, radiologically confirmed intestinal stenosis, or massive ascites (beyond the pelvic cavity) received CPT-11 alone (Cohort I). The remaining 87 patients received weekly PAC as they did not meet the safety criteria for administration of CPT-11 (Cohort P). Patients were selected according to the following criteria: i) histologically confirmed GC with metastatic or recurrent disease; ii) an Eastern Cooperative Oncology Group (ECOG) PS of 0-2; iii) failure of prior chemotherapy with a fluoropyrimidine-based regimen, either in an adjuvant setting or for metastatic disease; iv) age $\geq 18$ years; v) adequate function of bone marrow and preserved organ function; vi) no synchronous double cancer or other serious disease; and vii) availability of written informed consent prior to administration of the treatment.

Treatment. In Cohort I, patients received infusional $150 \mathrm{mg} / \mathrm{m}^{2}$ CPT-11 on days 1 and 15 every 4 weeks following pre-medication with an intravenous $5-\mathrm{HT}_{3}$ blocker and dexamethasone. In Cohort P, patients received infusional $80 \mathrm{mg} / \mathrm{m}^{2} \mathrm{PAC}$ on days 1,8 , and 15 every 4 weeks after pre-medication with an intravenous $5-\mathrm{HT}_{3}$ blocker. Diphenhydramine, dexamethasone and ranitidine were also administered to prevent hypersensitivity reactions. Chemotherapy was continued until the event of disease progression, unacceptable toxicities, or the patient's refusal of treatment.

Response and toxicity evaluation. Each patient underwent CT scan after every two cycles of therapy to document the extent of disease and evaluate response to treatment. Objective responses for measurable lesions were assessed according to the guidelines of the Response Evaluation Criteria in Solid Tumors
Committee (RECIST 1.0). Symptomatic toxicity and laboratory data were noted every 2 weeks at the outpatient department. Toxicities were assessed using the Common Toxicity Criteria for Adverse Events, version 3.0 (CTCAE v.3.0). Dose reduction and treatment delays were advocated according to the extent of hematological and non-hematological toxicities.

Statistical analysis. Progression-free survival was calculated from initiation of the second-line treatment until the time of progression occurred, the patient succumbed to any cause, or when neither of these events occurred, the date of the last follow-up. Overall survival was calculated from the time the second-line treatment commenced to the date of death or the last follow-up visit. Survival curves were obtained using the Kaplan-Meier method. Univariate analysis of OS and PFS was performed using the log-rank test. Statistical analyses were performed using SPSS (SPSS Inc., Chicago, IL, USA). $\mathrm{P}$-values were two-sided; $\mathrm{P}<0.05$ was regarded as statistically significant.

\section{Results}

Patient characteristics. Table I shows the patient characteristics. The median age of the patients was 61 years (range 19-82) in Cohort I and 62 years (range 28-73) in Cohort P, and the majority of the study population was male (64.2\%). A total of $164(91.6 \%)$ patients had an ECOG PS of 0 or 1. One of the 92 (1.1\%) patients in Cohort I and 14 of the 87 (16.1\%) patients in Cohort P had a PS of 2. Primary lesions were present in 41 of 92 (44.6\%) patients in Cohort I and in 43 of 87 (49.4\%) patients in Cohort P. The patients had previously received chemotherapy with fluoropyrimidine-based regimens containing S-1, S-1 plus CDDP, capecitabine plus CDDP, or 5-FU plus methotrexate. Tumor response was evaluated in 125 of the 179 (69.8\%) patients with measurable target lesions. The lymph nodes (Cohorts I and P: 44.6 and 39.1\%, respectively), peritoneum (Cohorts I and P: 39.1 and $85.1 \%$, respectively) and the liver (Cohorts I and P: 39.1 and 24.1\%, respectively) were the most common metastatic sites. Among the 179 patients, histological type was intestinal in $50(27.9 \%)$ patients and diffuse in $129(72.1 \%)$ patients, according to the Lauren classification. Chemotherapy was administered in a third-line setting in 72 of 92 (78.3\%) patients in Cohort I and in 15 of 87 (17.2\%) patients in Cohort P. Of the 72 patients who received third-line chemotherapy in Cohort I, 53 (73.6\%) patients received weekly PAC and 14 (19.4\%) patients received tri-weekly docetaxel (DOC).

Response and survival. No complete response was observed among the 179 patients, and overall response rate (ORR) was $6.5 \%$ in Cohort I and 9.8\% in Cohort P. Disease control (partial response and stable disease) rate (DCR) was $43.5 \%$ in Cohort I and $54.9 \%$ in Cohort P. Median PFS was comparable between 2.6 months (95\% CI, 2.2-3.0) in Cohort I and 2.8 months (95\% CI, 2.5-3.0) in Cohort $\mathrm{P}(\mathrm{P}=0.812)$ (Fig. 1). Median OS was 9.8 months (95\% CI, 7.8-11.8) in Cohort I and 4.9 months (95\% CI, 3.8-5.9) in Cohort $\mathrm{P}(\mathrm{P}<0.0001)$ (Fig. 2). A significant difference was observed in OS between Cohort I and Cohort P.

Adverse events. Table II shows the adverse effects observed in this study. The most frequent grade $3 / 4$ adverse hematological 
Table I. Patient characteristics $(\mathrm{n}=177)$.

\begin{tabular}{|c|c|c|}
\hline Characteristics & Irinotecan, $\mathrm{n}(\%)(\mathrm{n}=92)$ & Paclitaxel, n (\%) $(\mathrm{n}=87)$ \\
\hline \multicolumn{3}{|l|}{ Gender } \\
\hline Male & $67(72.8)$ & $48(55.2)$ \\
\hline Female & $25(27.2)$ & $39(44.8)$ \\
\hline Median age (range) & $61(19-82)$ & $62(28-73)$ \\
\hline \multicolumn{3}{|l|}{ ECOG PS } \\
\hline 0 & $73(79.3)$ & $37(42.5)$ \\
\hline 1 & $18(19.6)$ & $36(41.4)$ \\
\hline 2 & $1 \quad(1.1)$ & $14(16.1)$ \\
\hline Previous gastrectomy (+) & $51(55.4)$ & $44(50.6)$ \\
\hline \multicolumn{3}{|l|}{ Histological type ${ }^{a}$} \\
\hline Intestinal & $37(40.2)$ & $13(14.9)$ \\
\hline Diffuse & $55(59.8)$ & $74(85.1)$ \\
\hline Target lesions (+) & $74(80.4)$ & $35(83.3)$ \\
\hline Massive ascites (+) & $0 \quad(0.0)$ & $33(37.9)$ \\
\hline \multicolumn{3}{|l|}{ No. of involved organs } \\
\hline 1 & $37(40.2)$ & $30(34.5)$ \\
\hline$\geq 2$ & $55(59.8)$ & $57(65.5)$ \\
\hline \multicolumn{3}{|l|}{ Metastatic sites } \\
\hline Lymph nodes & $41(44.6)$ & $34(39.1)$ \\
\hline Peritoneum & $36(39.1)$ & $74(85.1)$ \\
\hline Liver & $36(39.1)$ & $21(24.1)$ \\
\hline Lung & $6 \quad(6.5)$ & $2(2.3)$ \\
\hline Bone & $1 \quad(1.1)$ & $9(10.3)$ \\
\hline Ovary & $5 \quad(5.4)$ & $11(12.6)$ \\
\hline Adrenal gland & $0 \quad(0.0)$ & $4(4.6)$ \\
\hline Portal embolism & $2(2.2)$ & $2(2.3)$ \\
\hline Other & $1 \quad(1.1)$ & $3(3.4)$ \\
\hline \multicolumn{3}{|l|}{ Prior chemotherapy } \\
\hline S-1 & $21(22.8)$ & $44(50.6)$ \\
\hline S-1 plus CDDP & $55(59.8)$ & $21(24.1)$ \\
\hline CAP plus CDDP & $4 \quad(4.3)$ & $2(2.3)$ \\
\hline 5-FU plus MTX & $2(2.2)$ & $14(16.1)$ \\
\hline Other & $0 \quad(0.0)$ & $2(2.3)$ \\
\hline Adjuvant S-1 & $10(10.9)$ & $4 \quad(4.6)$ \\
\hline Third-line chemotherapy & $72(78.2)$ & $15(17.2)$ \\
\hline Weekly PAC & $53(57.6)$ & $0 \quad(0.0)$ \\
\hline Tri-weekly DOC & $14(15.2)$ & $0 \quad(0.0)$ \\
\hline 5-FU plus MTX & 1 (1.1) & $5 \quad(5.7)$ \\
\hline CPT-11 & $0 \quad(0.0)$ & $6 \quad(6.9)$ \\
\hline Other & $4(4.3)$ & 4 (4.6) \\
\hline
\end{tabular}

aLauren classification. CDDP, cisplatin; CAP, capecitabine; 5-FU, fluorouracil; MTX, methotrexate; PAC, paclitaxel; DOC, docetaxel.

event was neutropenia, which was observed in 7 of $92(7.6 \%)$ patients in Cohort I and in 13 of $87(14.9 \%)$ patients in Cohort P. Leukopenia was found in 4 of 92 (4.3\%) patients in Cohort I and in 4 of 87 (4.6\%) patients in Cohort P. Anemia was noted in 3 of $92(3.3 \%)$ patients in Cohort I and in 10 of $87(11.5 \%)$ patients in Cohort P. Septic shock occurred in one (1.1\%) patient, who died within 30 days of the last administration of the CPT-11 alone regimen. The most common grade 


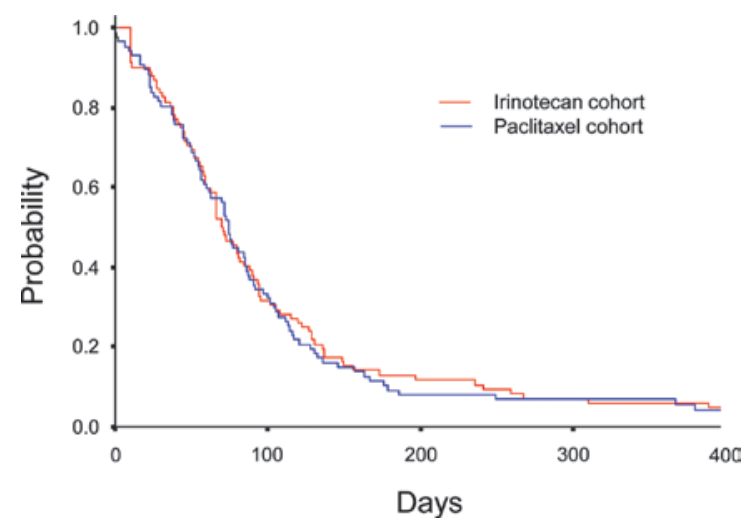

Figure 1. Progression-free survival from initiation of the second-line chemotherapy.

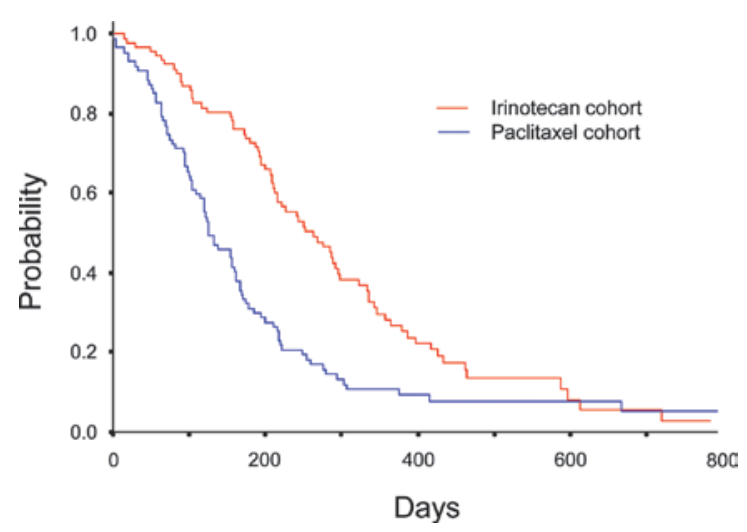

Figure 2. Overall survival from initiation of second-line chemotherapy.

Table II. Frequency of grade 3/4 adverse events.

Adverse events (AEs) Irinotecan, $\mathrm{n}(\%)(\mathrm{n}=92)$ Paclitaxel, n (\%) (n=87)

$\begin{array}{lrr}\text { Hematological AEs } & & \\ \text { Leukopenia } & 4(4.3) & 4(4.6) \\ \text { Neutropenia } & 7(7.6) & 13(14.9) \\ \text { Anemia } & 3(3.3) & 10(11.5) \\ \text { Thrombocytopenia } & 1(1.1) & 1(1.1) \\ \text { Febrile neutropenia } & 1(1.1) & 2(2.3) \\ \text { Non-hematological AEs } & & \\ \text { Anorexia } & 5(5.4) & 4(4.6) \\ \text { Nausea } & 2(2.2) & 0(0.0) \\ \text { Fatigue } & 1(1.1) & 3(3.4) \\ \text { Diarrhea } & 3(3.3) & 1(1.1) \\ \text { Hyponatremia } & 0(0.0) & 4(4.6) \\ \text { Hyperkalemia } & 1(1.1) & 1(1.1) \\ \text { Hypokalemia } & 0(0.0) & 3(3.4) \\ \text { Increased transaminase } & 0(0.0) & 4(4.6) \\ \text { Neuropathy (sensory) } & 0(0.0) & 2(2.3) \\ \text { Treatment-related deaths } & 1(1.1) & 0(0.0)\end{array}$

3/4 adverse non-hematological event was anorexia, which was found in 5 of 92 (5.4\%) patients in Cohort I and in 4 of 87 (4.6\%) patients in Cohort P.

\section{Discussion}

First-line chemotherapy is considered to confer a survival benefit in the treatment of advanced GC. However, optimal second-line chemotherapy contributes to the favorable OS observed in first-line treatment. Few well-designed, randomized trials of chemotherapy in a second-line setting have been conducted and the optimal treatment regimen following the failure of first-line chemotherapy remains to be determined (16). The results of the AIO phase III trial suggest that CPT-11 is a key novel agent in a second-line setting (11).

In advanced colorectal cancer, the availability of the three active cytotoxic agents of FU-leucovorin, CPT-11 and oxaliplatin during the treatment course is crucial as it maximizes the
OS of patients (17). In the treatment of advanced GC, combination regimens using FU with CDDP are currently regarded as standard first-line therapy. However, the importance to OS of the availability of two active cytotoxic agents, CPT-11 and the taxane agent $\mathrm{PAC}$ or DOC, during further treatment remains to be determined. CPT-11 often cannot be used following the failure of second-line treatment since severe toxicities may occur, in that GC progressively develops clinical symptoms of intestinal stenosis, massive ascites and obstructive jaundice due to dissemination. Therefore, CPT-11 is the treatment of choice in a second-line setting, unless contraindicated due to peritoneal metastasis or inadequate liver function.

Based on reports of its activity against other types of cancer $(18,19)$, PAC, a taxane agent, is frequently used in a fractionated weekly schedule at $80 \mathrm{mg} / \mathrm{m}^{2}$ in a second-line or further setting for GC. In previous phase II trials, weekly PAC administration was found to be active against GC with peritoneal metastasis $(14,15)$. Moreover, it has been suggested 
that the regimen is feasible, safe and shows consistent activity against heavily treated GC in a second-line or further setting $(12,20)$. Neutropenia (14.9\%), anemia (10\%), leukopenia (4\%), and anorexia (4\%) were the most common grade $3 / 4$ adverse events observed in this study, occurring less frequently than in previous reports. In Cohort $\mathrm{P}$ patients who were unable to receive $\mathrm{CPT}-11$ in a second-line setting, the median PFS was 77 days and median survival time was 136 days from initiation of PAC. This supports the feasibility of using PAC in patients contraindicated for CPT-11 due to evidence of peritoneal metastasis.

In this retrospective study, ORR and DCR in Cohort I were 6.5 and $43.5 \%$, respectively, compared with 9.8 and $54.9 \%$, respectively, in Cohort P. The median PFS (Cohort I vs. P, 2.6 vs. 2.8 months) did not differ between the two treatment cohorts (HR, 0.97; 95\% CI, 0.72-1.30; P=0.812). However, the median OS (Cohort I vs. P, 9.8 vs. 4.9 months) differed significantly between the two treatment cohorts (HR, 0.50; 95\% CI, 0.36-0.69; P<0.0001). PFS in Cohort I was comparable with that in Cohort $\mathrm{P}$, but OS in Cohort I was much longer. Third-line chemotherapy was administered in 72 of 92 (78.3\%) patients in Cohort I and in 15 of 87 (17.2\%) patients in Cohort P. The high frequency of third-line chemotherapy in Cohort I may explain the discrepancy in OS between the two cohorts. Among the 72 patients who received third-line chemotherapy in Cohort I, 53 (73.6\%) received weekly PAC and $14(19.4 \%)$ received tri-weekly DOC. This course of treatment indicates that availability of CPT-11 in a second-line setting prolongs the survival of patients with advanced GC.

It is difficult to confirm the optimal second-line regimen from the results of the present study, and it appears that in the course of treatment, the availability of the four active cytotoxic agents, FU, CDDP, CPT-11 and the taxane agent PAC or DOC, prolong the lives of patients with advanced GC. However, the availability of CPT-11 in a second-line setting, in particular, appears to beneficial in terms of survival. Although this was a retrospective study, the results provide evidence that the availability of CPT-11 in a second-line setting is beneficial to advanced GC patients in whom fluoropyrimidine-based firstline chemotherapy proved unsuccessful.

\section{References}

1. Ferlay J, Shin HR, Bray F, Forman D, Mathers C and Parkin DM: Estimates of worldwide burden of cancer in 2008: GLOBOCAN 2008. Int J Cancer: June 17, 2010 (Epub ahead of print).

2. Murad AM, Santiago FF, Petroianu A, Rocha PR, Rodrigues MA and Rausch M: Modified therapy with 5-fluorouracil, doxorubicin, and methotrexate in advanced gastric cancer. Cancer 72 : 37-41, 1993.

3. Pyrhönen S, Kuitunen T, Nyandoto P and Kouri M: Randomised comparison of fluorouracil, epidoxorubicin and methotrexate (FEMTX) plus supportive care with supportive care alone in patients with non-resectable gastric cancer. $\mathrm{Br} \mathrm{J}$ Cancer 71: 587-591, 1995

4. Wagner AD, Grothe W, Haerting J, Kleber G, Grothey A and Fleig WE: Chemotherapy in advanced gastric cancer: a systematic review and meta-analysis based on aggregate data. J Clin Oncol 24: 2903-2909, 2006.
5. Vanhoefer U, Rougier $\mathrm{P}$, Wilke $\mathrm{H}$, et al: Final results of a randomized phase III trial of sequential high-dose methotrexate, fluorouracil, and doxorubicin versus etoposide, leucovorin, and fluorouracil versus infusional fluorouracil and cisplatin in advanced gastric cancer: A trial of the European Organization for Research and Treatment of Cancer Gastrointestinal Tract Cancer Cooperative Group. J Clin Oncol 18: 2648-2657, 2000.

6. Van Cutsem E, Moiseyenko VM, Tjulandin S, et al: Phase III study of docetaxel and cisplatin plus fluorouracil compared with cisplatin and fluorouracil as first-line therapy for advanced gastric cancer: a report of the V325 Study Group. J Clin Oncol 24: 4991-4997, 2006

7. Cunningham D, Starling N, Rao S, et al; Upper Gastrointestinal Clinical Studies Group of the National Cancer Research Institute of the United Kingdom: Capecitabine and oxaliplatin for advanced esophagogastric cancer. N Engl J Med 358: 36-46, 2008.

8. Koizumi W, Narahara H, Hara T, et al: S-1 plus cisplatin versus S-1 alone for first-line treatment of advanced gastric cancer (SPIRITS trial): a phase III trial. Lancet Oncol 9: 215-221, 2008.

9. Boku N, Yamamoto S, Fukuda H, et al: Fluorouracil versus combination of irinotecan plus cisplatin versus S-1 in metastatic gastric cancer: a randomised phase 3 study. Lancet Oncol 10: 1063-1069, 2009.

10. Ajani JA, Rodriguez W, Bodoky G, et al: Multicenter phase III comparison of cisplatin/S-1 with cisplatin/infusional fluorouracil in advanced gastric or gastroesophageal adenocarcinoma study: the FLAGS trial. J Clin Oncol 28: 1547-1553, 2010.

11. Thuss-Patience PC, Kretzschmar A, Deist T, et al: Irinotecan versus best supportive care (BSC) as second-line therapy in gastric cancer. A randomized phase III study of the Arbeitsgemeinschaft Internistische Onkologie (AIO). J Clin Oncol 27 (Suppl 15): abs. 4540,2009

12. Hironaka S, Zenda S, Boku N, Fukutomi A, Yoshino T and Onozawa Y: Weekly paclitaxel as second-line chemotherapy for advanced or recurrent gastric cancer. Gastric Cancer 9: 14-18, 2006.

13. Kodera Y, Ito S, Mochizuki Y, et al: A phase II study of weekly paclitaxel as second-line chemotherapy for advanced gastric Cancer (CCOG0302 study). Anticancer Res 27: 2667-2671, 2007.

14. Yamada Y, Shirao K, Ohtsu A, et al: Phase II trial of paclitaxel by three-hour infusion for advanced gastric cancer with short premedication for prophylaxis against paclitaxel-associated hypersensitivity reactions. Ann Oncol 12: 1133-1137, 2001.

15. Yamaguchi K, Tada M, Horikoshi N, et al: Phase II study of paclitaxel with 3-h infusion in patients with advanced gastric cancer. Gastric Cancer 5: 90-95, 2002.

16. Wesolowski R, Lee C and Kim R: Is there a role for second-line chemotherapy in advanced gastric cancer? (Review). Lancet Oncol 10: 903-912, 2009.

17. Grothey A, Sargent D, Goldberg RM and Schmoll HJ: Survival of patients with advanced colorectal cancer improves with the availability of fluorouracil-leucovorin, irinotecan, and oxaliplatin in the course of treatment. J Clin Oncol 22: 1209-1214, 2004.

18. Rosenberg P, Andersson H, Boman K, et al: Randomized trial of single agent paclitaxel given weekly versus every three weeks and with peroral versus intravenous steroid premedication to patients with ovarian cancer previously treated with platinum. Acta Oncol 41: 418-424, 2002.

19. Sparano JA, Wang M, Martino S, et al: Weekly paclitaxel in the adjuvant treatment of breast cancer. N Engl J Med 358: 1663-1671, 2008.

20. Shimoyama R, Yasui H, Boku N, et al: Weekly paclitaxel for heavily treated advanced or recurrent gastric cancer refractory to fluorouracil, irinotecan, and cisplatin. Gastric Cancer 12: 206-211, 2009. 
